\title{
Review of "Health literacy and public health: a conceptual paper"
}

\author{
Giorgio Bedog $\mathrm{ni}^{1}$ \\ 1 Fondazione Italiana Fegato ONLUS
}

Disclaimer: I am a Qeios co-founder, an increasingly strong believer in Open Science, and a friend of the first Author.

Guglielmo, thank you for this thought-provoking paper on health literacy. I learnt a lot from it.

I am wondering whether Qeios can help us to reach some of the goals identified by Gazmararian et al. (2005):

$=>1$. Define what is a "health literate population";

2. Develop studies and tools to assess the health literacy levels of a population;

3. Critically evaluate the efforts that healthcare professionals spend in communication;

4. Enhance healthcare professionals' listening and communication skills;

5. Accept that, except for health literacy, having strong theoretical basis on health topics is necessary but not enough in order to maintain and promote healthy behaviours;

$=>6$. Develop extensive collaborations between public health and other professionals to achieve this goal.

=> means: here, in my opinion, Qeios can help.

Being based on Definitions, Qeios will help "by definition" with goal \# 1!

As goal \#6 is concerned, we hope that Qeios will stimulate the collaboration of researchers with different disciplinary backgrounds.

We are very open to the input of the Qeios community on this matter! 